\title{
Transverse Impact of a Linear Three-Element Spring and Dashpot Model Filament: Theory
}

\author{
Jack C. Smith \\ Institute for Materials Research, National Bureau of Standards, Washington, D.C. 20234
}

(July 7, 1966)

\begin{abstract}
The mathematics of wave propagation in a viscoelastic filament subjected to constant velocity transverse impact is discussed. The equations governing the stress-strain-time behavior are assumed to be those for a linear model consisting of a spring coupled in parallel with a spring and dashpot in series. The nature of the solution is discussed, and a method is described for calculating the configuration, and the stress, strain, and particle velocity distributions along the impacted filament. The method used consists of an integration along the characteristics of the system of differential equations describing the problem.
\end{abstract}

Key Words: Characteristics, impact, linear viscoelasticity, partial differential equations, stress waves, wave propagation.

\section{Introduction}

When a flexible filament is struck transversely by a high velocity projectile, strain waves and waves of transverse motion are generated. The associated displacement of filament material can be recorded by high speed photography, and the resulting data can be analyzed to obtain the stress-strain, creep, and stress relaxation behavior of the material under impact conditions. Research of this type has been described by Petterson et al., $[1,2,3]^{1}$ and Smith et al., $[4,5,6]$.

A theory originated by Rachmatulin [7], Taylor $[8,9]$, and von Karman [10], and modified by Smith et al., $[11,12]$ has been used to analyze the experimental data. This theory, however, requires additional modification in order to account adequately for viscoelastic effects. Malvern [13, 14] has helped in this respect by proposing a simple rate-dependent constitutive equation for approximating these effects, and has used it in treating the longitudinal impact problem. Further insight may be gained through the work of Lee and Kanter [15], Glauz and Lee [16], Morrison [17], and Smith [18] who have investigated the behavior of various linear spring and dashpot models subjected to longitudinal impact.

Transverse impact of materials having rate-dependent constitutive equations has received less attention. Cristescu [19, 20, 21, 22, 23] has discussed

\footnotetext{
${ }^{1}$ Figures in brackets indicate the literature references at the end of this paper.
}

various aspects of the problem of strain wave propagation and transverse wave motion in strings, including some strings with stress-strain-time behavior given by rate dependent constitutive equations. For some cases solutions calculated by a digital computer were obtained by integration along the characteristics of the system of differential equations describing the problem, but usually only continuous solutions were considered.

The problem of transverse impact at constant velocity involves a solution in which the strain is discontinuous at the strain wave front, and the slope of the filament is discontinuous at the front of a wave of transverse motion. This latter problem will be discussed here, and a solution obtained by an extension of the methods of Cristescu. The understanding gained is expected to be of use in the design and analysis of future experiments.

\section{The Model}

The constitutive equation governing the stressstrain-time behavior of the filament is that for the three-element linear spring and dashpot model depicted in figure 1. The longitudinal impact behavior of this model has been treated previously by Morrison [17] and Smith [18]. In the model the Hooke's law constants of the two springs are $g_{1}$ and $g_{2}$, and the viscosity constant of the dashpot $\gamma_{1} . T$ is the total 


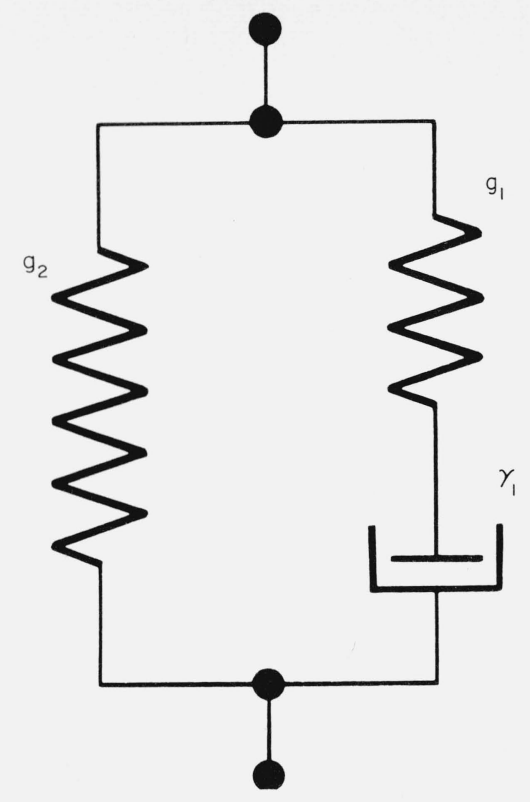

FIGURE 1. Linear spring and dashpot model consisting of two springs and one dashpot.

force, $\epsilon$ the strain, and $t$ the time. The constitutive equation is

$$
\left(g_{1}+g_{2}\right) \frac{\partial \epsilon}{\partial t}+\frac{g_{1} g_{2}}{\gamma_{1}} \epsilon=\frac{\partial T}{\partial t}+\frac{g_{1}}{\gamma_{1}} T .
$$

It is more convenient to represent the model in terms of other parameters. Thus let $g_{1}=\lambda g, g_{2}=$ $(1-\lambda) g, \gamma_{1}=\lambda g \tau$. The parameter $\tau=\gamma_{1} / g_{1}$ is the relaxation time of the Maxwell element constituting one branch of the model, and $\lambda$ is a dimensionless parameter that takes on values between 0 and 1 . When $\lambda=0$ the model degenerates into a spring of modulus $g$, and when $\lambda=1$ the model becomes a Maxwell element. For practical applications a value of $\lambda$ of approximately 0.1 coupled with a relaxation time $\tau$ less than the observation time following an impact is of interest. Such a model would be mostly springlike in its behavior, but would exhibit noticeable viscoelastic effects shortly after impact. Another convenient parameter is $c=\sqrt{g / m}$, the velocity at which a strain wave front propagates in the filament. The quantity $m$ is the mass per unit length of the unstrained filament. Equation (1) expressed in the parameters $\tau, \lambda, c$ becomes

$$
m c^{2} \frac{\partial \epsilon}{\partial t}+\frac{(1-\lambda) m c^{2}}{\tau} \epsilon=\frac{\partial T}{\partial t}+\frac{1}{\tau} T .
$$

\section{Formulation of the Problem}

In the laboratory or observer's coordinate system, let $x^{\prime}$ and $y^{\prime}$ denote the horizontal and vertical coordi- nates respectively. At time $t=0$ let the filament at $x^{\prime}=0$ be impacted at constant velocity $V$ in the $y^{\prime}$ direction. The motion of the filament is to be described in terms of a Lagrangian coordinate system; i.e., a system of $x$ coordinates fixed to the filament, moving and extending with it, but such that in the initial unstrained state the $x$ and $x^{\prime}$ coordinates of the filament coincide. The displacement of any point $x$ of the filament at time $t$ relative to its original position is given by $\xi(x, t)$ for horizontal displacements, and $\eta(x, t)$ for vertical displacements. The location in the laboratory coordinate system of a point $x$ on the filament is thus given by

$$
x^{\prime}=x+\xi(x, t), \quad y^{\prime}=\eta(x, t) .
$$

Consider an element of unstrained length $\Delta x$. After impact, this element is strained, and its new length measured in the laboratory coordinate system becomes $\Delta x \sqrt{(1+\partial \xi / \partial x)^{2}+(\partial \eta / \partial x)^{2}}$. The increase in length per unit length, or strain $\epsilon$, is thus

$$
\epsilon=\sqrt{(1+\partial \xi / \partial x)^{2}+(\partial \eta / \partial x)^{2}}-1 .
$$

The angle $\theta$ that the element makes with the horizontal is given by

$$
\cos \theta=\frac{1}{1+\epsilon}\left(1+\frac{\partial \xi}{\partial x}\right), \quad \sin \theta=\frac{1}{1+\epsilon} \frac{\partial \eta}{\partial x}
$$

so that the differential equations that govern the motion of the small element are

$$
\begin{aligned}
& m \frac{\partial^{2} \xi}{\partial t^{2}}=\frac{\partial}{\partial x}(T \cos \theta)=\frac{\partial}{\partial x}\left[\frac{T}{1+\epsilon}\left(1+\frac{\partial \xi}{\partial x}\right)\right] \\
& m \frac{\partial^{2} \eta}{\partial t^{2}}=\frac{\partial}{\partial x}(T \sin \theta)=\frac{\partial}{\partial x}\left[\frac{T}{1+\epsilon} \frac{\partial \eta}{\partial x}\right] .
\end{aligned}
$$

Equations (6a) and (6b) can be incorporated into a system of linear equations by introducing the horizontal and vertical components of the velocity (in $x^{\prime}$, $y^{\prime}$ space) of a point on the filament

$$
u=\partial \xi / \partial t, \quad v=\partial \eta / \partial t
$$

and the direction variables $\alpha, \beta$ defined by

$$
\alpha=1+\frac{\partial \xi}{\partial x}, \quad \beta=\frac{\partial \eta}{\partial x} .
$$

It is also helpful to introduce the flow or particle velocity $w$ of a point along the direction of the filament. An increment of this velocity $d w$ has horizontal and vertical components $d u$ and $d v$ respectively and thus satisfies the relation

$$
d w=d u \cos \theta+d v \sin \theta .
$$


The system of linear equations becomes

$$
\begin{aligned}
\frac{\partial u}{\partial t} & =\frac{\partial}{\partial x}\left[\frac{\alpha T}{m(1+\epsilon)}\right] \\
\frac{\partial v}{\partial t} & =\frac{\partial}{\partial x}\left[\frac{\beta T}{m(1+\epsilon)}\right] \\
\frac{\partial u}{\partial x} & =\frac{\partial \alpha}{\partial t} \\
\frac{\partial v}{\partial x} & =\frac{\partial \beta}{\partial t} \\
d \epsilon & =\frac{\alpha}{1+\epsilon} d \alpha+\frac{\beta}{1+\epsilon} d \beta \\
d w & =\frac{\alpha}{1+\epsilon} d u+\frac{\beta}{1+\epsilon} d v \\
m c^{2} \frac{\partial \epsilon}{\partial t}+ & \frac{(1-\lambda) m c^{2}}{\tau} \epsilon=\frac{\partial T}{\partial t}+\frac{1}{\tau} T .
\end{aligned}
$$

Equation (10e) is the differential form of eq (4).

The seven variables $u, v, w, \alpha, \beta, \epsilon$, and $T$ must satisfy the initial conditions: For $t=0, x>0$

$$
u=v=w=\beta=\epsilon=T=0, \alpha=1
$$

and the boundary conditions: For $t>0, x=0$

$$
u=0, v=V
$$

and for $t>0, x \rightarrow \infty$

$$
u=v=w=\beta=\epsilon=T=0, \alpha=1 .
$$

If conditions (12) are substituted into eq (10f), an additional boundary condition: For $t>0, x=0$

$$
w=a \text { constant }
$$

is obtained. The value of this constant velocity is attained at the instant of impact, before any creep or stress relaxation has occurred, and thus is the same as that given in the solution for the springlike filament discussed in the next section.

In addition to the initial and boundary conditions, certain compatibility conditions, to be given later, must be satisfied.

\section{Solution for the Case $\lambda=0$}

When $\lambda=0$ the model degenerates to a spring of Hooke's law constant $g$. The solution for this case, discussed in detail elsewhere [11] is illustrated in

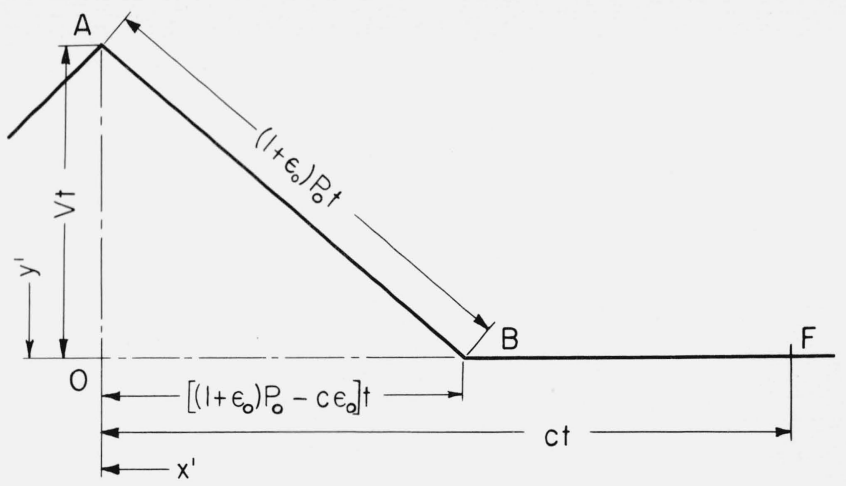

Figure 2. Configuration of a spring-like filament at time t after transverse impact.

The filament, originally horizontal, was struck with velocity $V$ in the vertical direction. After time $t$ the resulting strain wave propagating with velocity $c$ has a front at $\mathrm{F}$, and the filament becomes uniformly strained at strain $\epsilon_{0}$. The strained material between points $\mathrm{F}$ and $\mathrm{B}$ moves inward horizontally with velocity $-r \epsilon_{0}$, but at the transverse wave front, point B, this inward motion ceases. Material in the transverse wave moves vertically with velocity $V$. The transverse wave front moves with velocity $P_{0}$ relative to points fixed on the filament, but with velocity $\left(1+\epsilon_{0}\right) P_{0}-c \epsilon_{0}$ with respect to the laboratory $\left(x^{\prime}, y^{\prime}\right)$ coordinate system.

figure 2. The impact causes a wave of constant strain $\epsilon_{0}$ and constant tension $T_{0}$ to propagate along the filament at velocity $c$, so that at time $t$ the strain wave front has arrived at point $F$. In the wake of this wave front the strained material flows inward at velocity $-c \epsilon_{0}$. The increase in length of the filament is taken up by a wave of transverse motion, which at the time $t$ has a front at point $B$.

In the transverse wave the filament material moves in the $y^{\prime}$ direction at the impact velocity $V$, and there is no component of velocity in the $x^{\prime}$ direction. The profile of the wave is a straight line. The transverse wave front moves at velocity $P_{0}=\sqrt{T_{0} / m\left(1+\epsilon_{0}\right)}$ relative to the Lagragian coordinate system, so that the length of strained filament $\mathrm{AB}$ as measured in the $x^{\prime}, y^{\prime}$ system is equal to $\left(1+\epsilon_{0}\right) P_{0} T$, and the horizontal projection of this length is $\left[\left(1+\epsilon_{0}\right) P_{0}-c \epsilon_{0}\right] t$.

As $\mathrm{OAB}$ is a right triangle, the following relationship applies

$$
V^{2}=\left(1+\epsilon_{0}\right)^{2} P_{0}^{2}-\left[\left(1+\epsilon_{0}\right) P_{0}-c \epsilon_{0}\right]^{2} .
$$

If the mass per unit length $m$ and Hooke's law constant $g$ are known for the specimen, the velocities $c$ and $P_{0}$ can be calculated for any strain $\epsilon_{0}$, and upon substituting these values into eq (15), the value of $\epsilon_{0}$ corresponding to any transverse impact velocity $V$ can be found.

In the region between the strain wave front and the transverse wave front, the particle velocity $w$ and the horizontal velocity $u$ are both constant and equal to $-c \epsilon_{0}$. In the region of the transverse wave, $u=0$ but the velocity $w$ remains equal to $-c \epsilon_{0}$, even though in the $x^{\prime}, y^{\prime}$ coordinate system there is no observable flow of material longitudinally along the filament. This paradox can be explained by the following interpretation of the particle velocity $w$. Suppose that 
there is a second filament, inextensible and fixed to the impacted filament at a point a great distance in advance of the strain wave front. Before impact let the two filaments coincide with the inextensible filament ending at the point of impact, and after impact let the inextensible filament be constrained to move alongside the impacted filament. Under these conditions the point under impact will recede from the end of the inextensible filament with velocity $w$.

The solution for the limit case $\lambda=0$ can be stated briefly as follows. The quantities $w, \epsilon$, and $T$ are continuous between the point of impact and the strain wave front at $x=c t$, and have the following values:

$$
w=-c \epsilon_{0}, \epsilon=\epsilon_{0}, T=m c^{2} \epsilon_{0}
$$

where $\epsilon_{0}$ is found by solving eq (15). The quantities $u, v, \alpha, \beta$ are discontinuous at the transverse wave front $x=P_{0} t=t \sqrt{T_{0} / m\left(1+\epsilon_{0}\right)}$.

For $0 \leqslant x<P_{0} t$

$$
\begin{aligned}
& u=0, v=V \\
& \alpha=\left(1+\epsilon_{0}\right) \cos \theta=1+\epsilon_{0}-c \epsilon_{0} / P_{0}(17 \mathrm{a}, \mathrm{b}, \mathrm{c}, \mathrm{d}) \\
& \beta=\left(1+\epsilon_{0}\right) \sin \theta=-V / P_{0}
\end{aligned}
$$

and for $P_{0} t \leqslant x<c t$

$$
u=w=-c \epsilon_{0}, v=\beta=0, \alpha=1+\epsilon_{0} . \quad(18 \mathrm{a}, \mathrm{b}, \mathrm{c})
$$

The limit solution just presented indicates the type of solution to be sought for the general case where $\lambda>0$; namely, a solution in which $w, \epsilon$, and $T$ are continuous between the point of impact and the strain wave front, and $u, v, \alpha$, and $\beta$ are continuous only between the point of impact and the transverse wave front. A method for finding this solution by integrating along the characteristics of the system of differential equations will be developed in the succeeding sections.

\section{The Characteristics of the System of Equations}

If the variables $u, v, \alpha$, and $\beta$ are eliminated from eqs (10a) through $(10 \mathrm{~g})$ the following set of equations results. ${ }^{2}$

$$
\begin{aligned}
& \frac{\partial w}{\partial t}=\frac{1}{m} \frac{\partial T}{\partial x} \\
& \frac{\partial w}{\partial x}=\frac{\partial \epsilon}{\partial t} \\
& m c^{2} \frac{\partial \epsilon}{\partial t}+\frac{(1-\lambda) m c^{2}}{\tau} \epsilon=\frac{\partial T}{\partial t}+\frac{1}{\tau} T .
\end{aligned}
$$

$(19 \mathrm{a}, \mathrm{b}, \mathrm{c})$

${ }^{2}$ Equations (19a) through (19c) plus the boundary conditions of eqs (11) through (14 involving $w . \epsilon$. and $T$ are the same as the equations and boundary conditions for longitudinal impact at constant velocity $-c \epsilon_{0}$. The distribution of $w, \epsilon$. and $T$ can be calculated from the solutions for this case given by Morrison [17] and Smith [18]. However, the subsequent the solutions for this case given by Morrison $[17]$ and Smith [18]. However. the subsequent determination of values for $u, v$. $\alpha$, and $\beta$ from
These equations then can be arranged to obtain

$$
\begin{gathered}
m c\left(\frac{\partial w}{\partial t}+c \frac{\partial w}{\partial x}\right)-\left(\frac{\partial T}{\partial t}+c \frac{\partial T}{\partial x}\right)=\frac{1}{\tau}\left[T-(1-\lambda) m c^{2} \epsilon\right] \\
-m c\left(\frac{\partial w}{\partial t}-c \frac{\partial w}{\partial x}\right)-\left(\frac{\partial T}{\partial t}-c \frac{\partial T}{\partial x}\right)=\frac{1}{\tau}\left[T-(1-\lambda) m c^{2} \epsilon\right]
\end{gathered}
$$

For differentiations along curves of the families given by

$$
d x / d t= \pm c
$$

the expressions in parentheses may be replaced by total derivatives so that the equations reduce to: Along the curves given by $d x / d t=c$

$$
m c d w-d T=\left[T-(1-\lambda) m c^{2} \epsilon\right] d t / \tau
$$

and along the curves given by $d x / d t=-c$

$$
m c d w+d T=-\left[T-(1-\lambda) m c^{2} \epsilon\right] d t / \tau .
$$

The curves satisfying eq (20) are known as characteristics.

If $P=\sqrt{T / m(1+\epsilon)}$, eq (10a) can be expressed in the form

$$
\frac{\partial u}{\partial t}=P^{2} \frac{\partial \alpha}{\partial x}+\alpha \frac{\partial}{\partial x}\left(P^{2}\right)
$$

which can be combined with eq (10c) to obtain the expressions

$$
\begin{aligned}
& \frac{\partial u}{\partial t}+P \frac{\partial u}{\partial x}=P\left(\frac{\partial \alpha}{\partial t}+P \frac{\partial \alpha}{\partial x}\right)+\alpha \frac{\partial}{\partial x}\left(P^{2}\right) \\
& \frac{\partial u}{\partial t}-P \frac{\partial u}{\partial x}=-P\left(\frac{\partial \alpha}{\partial t}-P \frac{\partial \alpha}{\partial x}\right)+\alpha \frac{\partial}{\partial x}\left(P^{2}\right) .
\end{aligned}
$$

Along curves of the families given by

$$
d x / d t= \pm P
$$

these expressions reduce to

$$
\frac{d u}{d t}= \pm P \frac{d \alpha}{d t}+\alpha \frac{\partial}{\partial x}\left(P^{2}\right) .
$$

Similarly eqs (10b) and (10d) yield

$$
\frac{d v}{d t}= \pm P \frac{d \beta}{d t}+\beta \frac{\partial}{\partial x}\left(P^{2}\right) .
$$

Equations (23a) and (23b) can be combined to give: Along the curves given by $d x / d t=P$

$$
\beta d u-\alpha d v=P \beta d \alpha-P \alpha d \beta
$$


and along the curves given by $d x / d t=-P$

$$
\beta d u-\alpha d v=-P \beta d \alpha+P \alpha d \beta
$$

The curves satisfying eq (22) are additional characteristics.

Other equations holding along these characteristics can be found by the following manipulation involving eqs (19a) and (19b).

$\frac{\partial}{\partial x}\left(P^{2}\right)=\frac{(1+\epsilon)(\partial T / \partial x)-T(\partial \epsilon / \partial x)}{m(1+\epsilon)^{2}}$

$$
=\frac{1}{1+\epsilon}\left[\frac{1}{m} \frac{\partial T}{\partial x}-P^{2} \frac{\partial \epsilon}{\partial x}\right] .
$$

But along curves defined by $d x / d t=P$

$$
\frac{1}{m} \frac{\partial T}{\partial x}=\frac{\partial w}{\partial t}=\frac{d w}{d t}-P \frac{\partial w}{\partial x}
$$

and

$$
P^{2} \frac{\partial \epsilon}{\partial x}=P \frac{d \epsilon}{d t}-P \frac{\partial \epsilon}{\partial t}=P \frac{d \epsilon}{d t}-P \frac{\partial w}{\partial x}
$$

so that

$$
\frac{\partial}{\partial x}\left(P^{2}\right)=\frac{1}{1+\epsilon}\left[\frac{d w}{d t}-P \frac{d \epsilon}{d t}\right] .
$$

Thus along curves defined by $d x / d t=P$, eqs (23a) and (23b) reduce to

$$
\begin{aligned}
& (1+\epsilon)(d u-P d \alpha)=\alpha(d w-P d \epsilon) \\
& (1+\epsilon)(d v-P d \beta)=\beta(d w-P d \epsilon)
\end{aligned}
$$

Similarly along curves defined by $d x / d t=-P$

$$
\begin{aligned}
& (1+\epsilon)(d u+P d \alpha)=\alpha(d w+P d \epsilon) \\
& (1+\epsilon)(d v+P d \beta)=\beta(d w+P d \epsilon)
\end{aligned}
$$

Along characteristic curves in $x, t$ space the integral surface of the system of differential equations may have discontinuities. It should be noted that the characteristic curve $x=c t$ determines the discontinuous wave front for the stress, strain and particle velocity distributions, and the characteristic curve that passes through the origin and is a solution of the equation $d x / d t=P$, determines the front of the transverse wave where the quantities $u, v, \alpha$, and $\beta$ are discontinuous.

\section{Compatibility Conditions}

An obvious requirement of the solution is that the displacements $\xi$ and $\eta$ be continuous at all points along the filament. The partial derivatives of these quan- tities with respect to $x$ and $t$, however, are not necessarily continuous at the strain wave front and at the transverse wave front. At these places of discontinuity, according to the principle of kinematic compatibility [24], the following relations hold:

$$
\begin{aligned}
& {\left[\frac{\partial \xi}{\partial x}\right] \frac{d x}{d t}+\left[\frac{\partial \xi}{\partial t}\right]=0} \\
& {\left[\frac{\partial \eta}{\partial x}\right] \frac{d x}{d t}+\left[\frac{\partial \eta}{\partial t}\right]=0}
\end{aligned}
$$

where the bracketed quantities represent jumps in value occasioned by the passage of a wave front at the point under consideration. For instance, at the point $x$ the expression $[\epsilon]$ is defined as

$$
[\epsilon]=\lim _{\delta \rightarrow 0}\{\boldsymbol{\epsilon}(x, t+\delta)-\boldsymbol{\epsilon}(x, t)\} .
$$

The strain wave front velocity is greater than the transverse wave front velocity. Therefore at the strain wave front, where $d x / d t=c$, it follows that $\eta=0$ and, from eq (4), $\partial \xi / \partial x=\epsilon$. Equation (27a) then yields the relation

$$
[\epsilon]=-\frac{1}{c}[w] .
$$

Similarly at the transverse wave front, where $d x / d t=P$, eqs (27a) and (27b) yield

$$
\begin{array}{r}
{[\alpha-1]=-\frac{1}{P}[u]} \\
{[\beta]=-\frac{1}{P}[v]}
\end{array}
$$

As $[\alpha-1]=\alpha-1-\epsilon, \quad[u]=u-w, \quad[\beta]=\beta, \quad$ and $[v]=v$, where $\alpha, u, \beta$, and $v$ refer to values in the wake of the wave front, eqs (29a) and (29b) can be expressed more conveniently as

$$
\begin{aligned}
& \alpha=1+\epsilon-u / P+w / P \\
& \beta=-v / P
\end{aligned}
$$

Another compatibility condition is obtained as follows. Consider a point $x$ on the filament. Just before arrival of the strain wave front at time $t$, the tension $T$ and particle velocity $w$ are both zero. At a slightly later time $t+\Delta t=t+\Delta x / c$, the tension is $-\Delta x(\partial T / \partial x)$ and the particle velocity is $(\Delta x / c)(\partial w / \partial t)$. Thus the jump relations for $T$ and $w$ are $[T]=-\Delta x(\partial T / \partial x)$ and $[w]=(\Delta x / c)(\partial w / \partial t)$. Application of eq (19a) then yields the condition of dynamic compatibility

$$
[T]=-m c[w] .
$$




\section{Values of the Tension, Strain, and Particle Velocity at the Strain Wave Front}

Equation (2la) is a differential relation involving the tension $T$, strain $\epsilon$, and particle velocity $w$, that holds at the strain wave front. Hence if values of $T$ and $w$ from eqs (28) and (31) are substituted into this relation, it becomes $2 d \epsilon=-(\lambda \epsilon / \tau) d t$, which when integrated yields

$$
\epsilon(c t, t)=\epsilon(0,0) e^{-(\lambda t / 2 \tau)} .
$$

Substituting this result into eqs (28) and (31) then gives

$$
\begin{aligned}
& w(c t, t)=-c \epsilon(0,0) e^{-(\lambda t / 2 \tau)} \\
& T(c t, t)=m c^{2} \epsilon(0,0) e^{-(\lambda t / 2 \tau)} .
\end{aligned}
$$

When $x=t=0$ the strain and transverse wave fronts coincide. If values at $t=x=0$ are designated by a zero subscript, the boundary conditions give $u_{0}=0$, $v_{0}=V$, and eqs $(30 \mathrm{a})$ and $(30 \mathrm{~b})$ reduce to

$$
\begin{aligned}
& \alpha_{0}=1+\epsilon_{0}-c \epsilon_{0} / P_{0} \\
& \beta_{0}=-V / P_{0}
\end{aligned}
$$

Thus eq (4) expressed as $\alpha_{0}^{2}+\beta_{0}^{2}=\left(1+\epsilon_{0}\right)^{2}$, can be put in the form $V^{2}=P_{0}^{2}\left(1+\epsilon_{0}\right)^{2}-\left[\left(1+\epsilon_{0}\right) P_{0}-c \epsilon_{0}\right]^{2}$. But $P_{0}=c \sqrt{\epsilon_{0} /\left(1+\epsilon_{0}\right)}$ so that

$$
V^{2} / c^{2}=\epsilon_{0}\left(1+\epsilon_{0}\right)-\left[\sqrt{\epsilon_{0}\left(1+\epsilon_{0}\right)}-\epsilon_{0}\right]^{2} .
$$

The value of $\epsilon_{0}$ to be used in eqs (32), (33), and (34) is obtained by solving eq (36).

\section{Integration Along the Characteristics}

A numerical solution to the system of differential eqs (10a) through $(10 \mathrm{~g})$ and the initial and boundary conditions (11) through (14) can be found by integrating along the characteristics of the system. The nature of the problem and the method of solution are easily understood from an inspection of figure 3 , in which some of the characteristics are plotted.

The straight line characteristics of positive slope are solutions of the equation $d x / d t=c$. Their slope is equal to $1 / c$. The other straight line characteristics completing the network are solutions of the equation $d x / d t=-c$. The characteristic passing through the origin, and having the equation $x=c t$ corresponds to the discontinuity marking the wave front of the strain, tension, and particle velocity distributions. Thus in the domain between this characteristic and the $x$ axis the strain $\epsilon$, tension $T$, and particle velocity $w$ are zero. Along the characteristic the values of $\epsilon, w$, and $T$ are given by eqs (32), (33), and (34). These equations thus

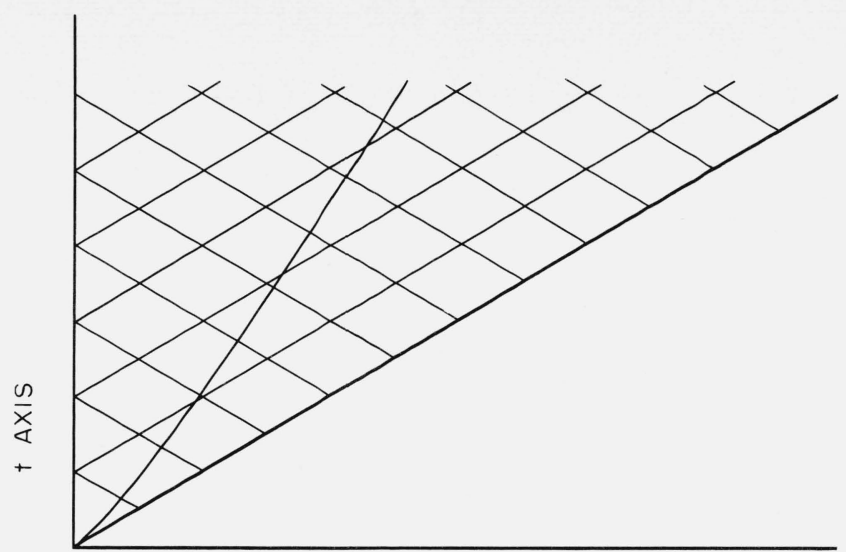

$x$ AXIS

FiguRE 3. A plot of the characteristics network.

The straight line characteristics of positive slope are solutions of the equation $d x / d t=c$ The straight line characteristics of negative slope are solutions of the equation $d x / d t=-c$. The straight line characteristic through the origin has the equation $x=c t$, or equation of the strain wave front. The curved characteristic passing through the origin is that solution of the equation $d x / d t=\sqrt{T / m(1+\epsilon)}$ that gives the location of the transverse wave front.

may be thought of as boundary conditions holding along the characteristic $x=c t$.

The curved line passing through the origin represents a solution of the characteristic equation $d x / d t=P$. The exact course of this characteristic is not known in advance, as $P$ is a function of $\epsilon$ and $T$. This characteristic corresponds to the front of the wave of transverse motion that propagates along the filament; thus in the domain between this characteristic and the characteristic $x=c t$ the velocity $u$ is equivalent to the velocity $w$, the direction variable $\alpha$ is equal to $1+\epsilon$, and the velocity $v$ and direction variable $\beta$ are equal to zero. The jump relations (30a) and (30b) holding along the transverse wave front characteristic constitute a second set of boundary conditions.

A third set of boundary conditions expressed by eqs (12a), (12b), and (14) holds along the time axis.

The problem consists in finding the values of $w$, $\epsilon$, and $T$ at all of the mesh points in the domain between and along the time axis and the characteristic $x=c t$, in finding the course of the transverse wave front characteristic, and in finding the values of $u$, $v, \alpha$, and $\beta$ at all of the mesh points between and along the time axis and the transverse wave front characteristic. The values of these quantities must satisfy the differential relations (10e), and (10f), the relation $(10 \mathrm{~g})$ holding along the line $x=a$ constant, the differential relations (2la), (2lb), (24a), (24b), (25a), (25b), (26a), and (26b) holding along the characteristics, and the boundary conditions.

A first step towards the solution consists in solving eq (36) for the initial strain $\epsilon_{0}$ at the point of impact. The values of $\epsilon, w$, and $T$ along the characteristic $x=c t$ are then given by eqs (32), (33), and (34).

Values of $\epsilon, w$, and $T$ are then found at the first mesh point on the time axis above the origin. Figure 4a 

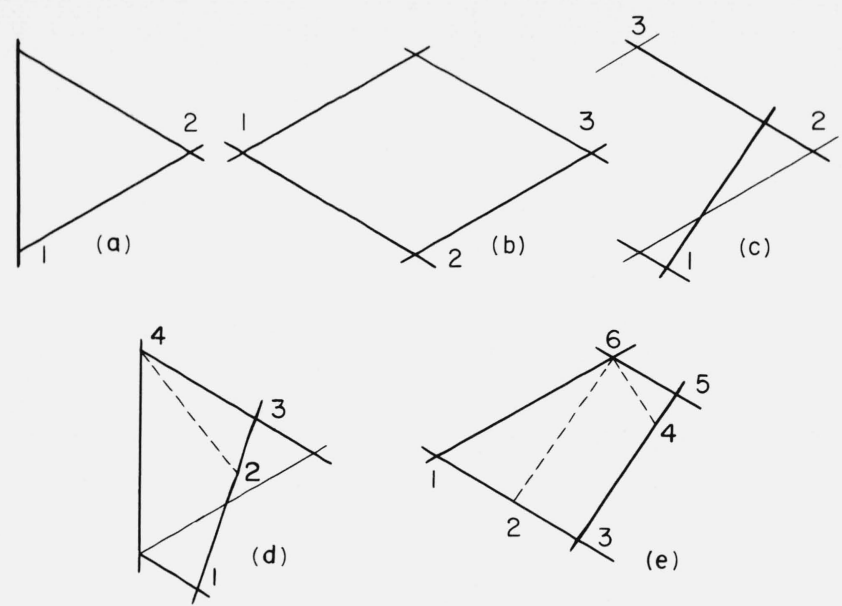

FIGURE 4. Mesh situations encountered during integration of the system of differential equations along the characteristics network.

depicts one of the triangular meshes lying along the $t$ axis. In the lowest mesh points the values of $\epsilon, w$, and $T$ are known, and from the boundary condition (14) $w$ is known to be constant along the $t$ axis. The strain $\epsilon$ and tension $T$ are found by solving eq (2lb), which holds along the characteristic of negative slope, and eq $(10 \mathrm{~g})$, which holds along the $t$ axis. These equations can be expressed in the form

$$
\begin{aligned}
& T-T_{2}=-\left[\bar{T}_{2}-(1-\lambda) m c^{2} E_{2}\right] \Delta t / \tau-m c\left(w_{1}-w_{2}\right) \\
& m c^{2}\left(\epsilon-\epsilon_{1}\right)-\left(T-T_{1}\right)=2\left[\bar{T}_{1}-(1-\lambda) m c^{2} E_{1}\right] \Delta t / \tau
\end{aligned}
$$

where $\Delta t=t-t_{2}$ and $2 \Delta t=t-t_{1}$. An iterative process is used in the solution. The quantities $\bar{T}_{1}, \bar{T}_{2}$, $E_{1}$, and $E_{2}$ are first set equal to $T_{1}, T_{2}, \epsilon_{1}$, and $\epsilon_{2}$, respectively, and approximate values are found for $T$ and $\epsilon$. The quantities $\bar{T}_{1}, \bar{T}_{2}, E_{1}$, and $E_{2}$ are then assigned the average values $\left(T+T_{1}\right) / 2,\left(T+T_{2}\right) / 2$. $\left(\epsilon+\epsilon_{1}\right) / 2$, and $\left(\epsilon+\epsilon_{2}\right) / 2$, respectively, and eqs (37a) and $(37 \mathrm{~b})$ are solved again for more accurate values of $T$ and $\epsilon$. Another iteration may be performed if desired.

Figure $4 \mathrm{~b}$ depicts a rhombus shaped mesh formed by the intersection of characteristics. If values of $\epsilon, w$, and $T$ are known at each of points 1,2 , and 3 the values of $\epsilon, w$, and $T$ at the vertex are found by solving the system of equations; eq (2la) which holds along the upper characteristic passing through point 1 , eq $(10 \mathrm{~g})$ which holds along the vertical line passing through point 2 , and eq $(2 \mathrm{lb}$ ) which holds along the upper characteristic passing through point 3 . The solution is found by an iterative process similar to that just described.

By applying successively the methods of solution associated with the mesh types depicted in figures $4 \mathrm{a}$ and $4 \mathrm{~b}$, values of $\epsilon, w$, and $T$ can be found at each of the mesh points in the domain between the $t$ axis and the characteristic $x=c t$.

The values of $\alpha$ and $\beta$ at the origin are next calculated from eqs (30a) and (30b). The values of $u$ and $v$ are already known from the boundary conditions. The solution for the mesh situation depicted in figure $4 \mathrm{c}$ can then be used to determine the course of the transverse wave front characteristic and the values of $\boldsymbol{\epsilon}, w, T, u, v, \alpha$, and $\beta$ at its intersections with the other characteristics. In figure $4 \mathrm{c}$ the quantities $\epsilon, w, T, u$, $v, \alpha$, and $\beta$ are known at point 1 , and the values of $\epsilon, w$, and $T$ are known at points 2 and 3 . The values of $\epsilon, w, T, u, v, \alpha$, and $\beta$ are required at the intersection of the transverse wave front characteristic with the characteristic passing through points 2 and 3 . As a first step $P$ is set equal to $\sqrt{T_{1} / m\left(1+\epsilon_{1}\right)}$ and the line $\left(x-x_{1}\right)=P\left(t-t_{1}\right)$ is drawn through point 1 to intersect the characteristic between points 2 and 3 . The values of $T$ and $\epsilon$ at the intersection are determined by interpolation. The quantity $P$ is then set equal to $\sqrt{\bar{T} / m(1+E)}$, where $\bar{T}$ and $E$ are the averages of $T$ and $T_{1}$, and $\epsilon$ and $\epsilon_{1}$ respectively. One or two iterations are performed to obtain more accurate values of $T$ and $\epsilon$ and the coordinates $(x, t)$ of the intersection. The value of $w$ at the intersection is then found by interpolation. The values of $u, v, \alpha$, and $\beta$ at the intersection are found by solving the set of four equations (30a), (30b), (25a), and (25b) expressed in the form

$$
\begin{aligned}
u-u_{1}-P\left(\alpha-\alpha_{1}\right) & =\frac{A}{1+E}\left[w-w_{1}-P\left(\epsilon-\epsilon_{1}\right)\right] \\
u+P \alpha & =w+P(1+\epsilon)
\end{aligned}
$$

$$
v-v_{1}-P\left(\beta-\beta_{1}\right)=\frac{B}{1+\epsilon}\left[w-w_{1}-P\left(\epsilon-\epsilon_{1}\right)\right]
$$

$$
v+P \beta=0 .
$$

In the solution process $A$ and $B$ are first set equal to $\alpha_{1}$ and $\beta_{1}$ respectively and in subsequent iterations set equal to the average values along the transverse wave front characteristic.

Solutions are needed for two additional mesh situations in order to find the values of $u, v, \alpha$, and $\beta$ at the mesh points between the $t$ axis and the transverse wave front characteristic. Figure $4 d$ depicts a mesh of the type found along the $t$ axis. The characteristic between points 1 and 3 may be either the transverse wave front characteristic or one of the solutions of the characteristic equation $d x / d t=c$. Usually the mesh is triangular in shape with point 1 lying on the $t$ axis, but sometimes when the transverse wave front characteristic has a steep slope, the mesh has the more general form shown. The values of $\epsilon, w, T, u, v, \alpha, \beta$ and the coordinates $x$ and $t$ are known at points 1 and 3 , and the values of $u, v, w, \epsilon$, and $T$ are known at point 4 . The values of $\alpha$ and $\beta$ at point 4 are required. As a first step the characteristic of slope $-1 / P$ is drawn 
between points 4 and 2 . In this construction iteration and interpolation processes are used to determine values of $P, \epsilon_{2}$, and $T_{2}$, and the coordinates $\left(x_{2}, t_{2}\right)$ of point 2. Interpolated values for $u_{2}, v_{2}, \alpha_{2}, \beta_{2}$, and $w_{2}$ are calculated next. The values of $\alpha$ and $\beta$ at point 4 are then obtained by solving eqs (26a) and (26b).

Figure 4e depicts a four-sided mesh in which the characteristic between points 3 and 5 may be either the transverse wave front characteristic or a solution of the characteristic equation $d x / d t=c$. Values of $u, v, w, \alpha, \beta, \epsilon$, and $T$ are known at points 1,3 , and 5 , and values of $\epsilon, w$, and $T$ are known at point 6 . The values of $u, v, \alpha$, and $\beta$ at point 6 are required. To find the solution lines of slope $+1 / P$ and $-1 / P$ are constructed to intersect at points 2 and 4 , respectively, and values of $u, v, w, \alpha, \beta, \epsilon$, and $T$ are determined at these points. The values of $u$ and $\alpha$ at point 6 are then obtained by solving eqs (25a) and (26a) expressed in the form

$$
\begin{aligned}
& u-u_{2}-P\left(\alpha-\alpha_{2}\right)=\frac{A}{1+E}\left[w_{6}-w_{2}-P\left(\epsilon_{6}-\epsilon_{2}\right)\right] \\
& u-u_{4}+P\left(\alpha-\alpha_{4}\right)=\frac{A}{1+E}\left[w_{6}-w_{4}+P\left(\epsilon_{6}-\epsilon_{4}\right)\right] .
\end{aligned}
$$

The values of $v$ and $\beta$ at point 6 are obtained similarly by solving eqs (25b) and (26b).

By systematically applying the methods of solution outlined for the five mesh types depicted in figure 4 , it is possible to calculate numerical values of $\epsilon, w, T$, $u, v, \alpha$, and $\beta$ at any distance $x$ along the filament, and at any time $t$.

In order to find the configuration of the filament in laboratory $\left(x^{\prime}, y^{\prime}\right)$ coordinates it is only necessary to calculate appropriate values of $\xi(x, t)$, and $\eta(x, t)$ by integrating $u(x, t)$ and $v(x, t)$ with respect to time. It is of special interest to perform this calculation in order to determine how much curvature is introduced into the transverse wave portion of the filament by the creep and stress relaxation processes that occur after impact.
The methods of calculation just described have been incorporated into a set of programs for use with a digital computer. Calculations have been made for various values of the propagation velocity $c$, impact velocity $V$, relaxation time $\tau$, and parameter $\lambda$ that approximate conditions realized in tests on real materials. It is planned to present some of the results of these calculations in a later publication.

\section{References}

[1] D. R. Petterson, G. M. Stewart, F. A. Odell, and R. C. Maheux, Textile Research J. 30, 411 (1960).

[2] D. R. Petterson and G. M. Stewart, Textile Research J. 30, 422 (1960).

[3] J. W. Jameson, G. M. Stewart, D. R. Petterson, and F. A. Odell, Textile Research J. 32, 858 (1962).

[4] J. C. Smith, C. A. Fenstermaker, and P. J. Shouse, Textile Research J. 33, 919 (1963).

[5] J. C. Smith, C. A. Fenstermaker, and P. J. Shouse, Textile Research J. 35, 743 (1965).

[6] C. A. Fenstermaker and J. C. Smith, Applied Polymer Symposia 1, 125 (1965).

[7] Kh. A. Rachmatulin, Prikl. Mat. i. Mech. 9, 91 (1945).

[8] G. I. Taylor, Scientific Papers of G. I. Taylor, Vol. 1, Paper No. 30, 456, Cambridge University Press (1958).

[9] G. I. Taylor, Scientific Papers of G. I. Taylor, Vol. 1, Paper No. 32, 467, Cambridge University Press (1958).

[10] T. von Karman and P. Duwez, J. Appl. Phys. 21, 987 (1950).

[11] J. C. Smith, F. L. McCrackin, and H. F. Schiefer, J. Res. NBS 60, 517 (1958); Textile Res. J. 28, 288 (1958).

[12] J. C. Smith, J. M. Blandford, and K. M. Towne, Textile Res. J. 32, 67 (1962).

[13] L. E. Malvern, J. Appl. Mech. 18, 203 (1951).

[14] L. E. Malvern, Quart. Appl. Math. 8, 405 (1951).

[15] E. H. Lee and I. Kanter, J. Appl. Phys. 24, 1115 (1953).

[16] R. D. Glauz and E. H. Lee, J. Appl. Phys. 25, 947 (1954).

[17] J. A. Morrison, Quart. Appl. Math. 14, 153 (1956).

[18] J. C. Smith, J. Appl. Phys. 37, 1697 (1966).

[19] N. Cristescu, Arch. Mech. Stos. 12, 597 (1960).

[20] N. Cristescu, J. Mech. Phys. Solids 9, 165 (1961).

[21] N. Cristescu, Arch. Mech. Stos. 15, 47 (1963).

[22] N. Cristescu, International Symposium on Stress Waves in Anelastic Solids, p. 118 (Springer Verlag, 1964).

[23] N. Cristescu, J. Mech. Phys. Solids 12, 269 (1964).

[24] A. G. Webster, Partial Differential Equations of Mathematical Physics, p. 283, 2d ed. (Dover, 1955).

(Paper 70B4-187) 